\title{
Optimizations of nonlinear kicker injection for synchrotron light sources
}

\author{
C. Sun, ${ }^{*}$ Ph. Amstutz, T. Hellert, S. C. Leemann, C. Steier, C. Swenson, and M. Venturini \\ Lawrence Berkeley National Laboratory, Berkeley, California, 94720, USA
}

(Received 4 June 2019; published 3 January 2020)

\begin{abstract}
The concept of using a single nonlinear kicker (NLK) to inject electron beams into a storage ring has been proposed and tested in several synchrotron radiation light source facilities. Different from pulsed dipole kicker magnets used in a conventional local-bump injection, the single nonlinear kicker provides a nonlinear distribution of magnetic fields which has a maximum value off axis where the injected beam arrives and a zero or near-zero value at the center where the stored beam passes by. Therefore, the injected beam will receive a kick from the NLK and lose its transverse momentum, and will be eventually captured by the storage ring. In the meantime the stored beam at the center will receive no kick or less kick, which significantly reduces the injection perturbations on the stored beam. In addition, the NLK injection requires less space for the kicker and removes the complications of synchronizing four pulsed kicker magnets. Because of these advantages, several light source facilities are either proposing or already using this NLK injection as a replacement of the conventional local-bump injection scheme. In this paper, we will discuss the working principal of this NLK injection, and use both Advanced Light Source and Advanced Light source Upgrade as examples to optimize the NLK injections. By optimizing the NLK design and injection conditions, we could achieve maximum injection efficiencies for both facilities with a large injected beam from the existing ALS booster.
\end{abstract}

DOI: 10.1103/PhysRevAccelBeams.23.010702

\section{INTRODUCTION}

For most existing or future synchrotron radiation light source facilities, the top-off operation is a standard mode of operation for a storage ring to provide a constant electron beam current to users without injection interruption [1]. This operation mode requires injecting a fresh electron beam into the storage ring frequently. The most commonly used injection method is a local bump injection where four pulsed kicker magnets are used to form a closed local bump around the injection point to reduce the separation between the injected beam and the stored beam. The injected beam will be eventually captured by the ring acceptance via synchrotron radiation damping. However, it remains technically challenging to perfectly match the four pulsed kickers to completely close the injection bump due to pulse jitters, shapes, and fields errors. The unclosed bump will cause the oscillation of the stored beam, therefore perturbing user experiments. To solve this problem, a new injection method using a single pulsed multipole magnet (PMM) has been proposed and tested at the Photon Factory

\footnotetext{
*ccsun@lbl.gov
}

Published by the American Physical Society under the terms of the Creative Commons Attribution 4.0 International license. Further distribution of this work must maintain attribution to the author(s) and the published article's title, journal citation, and DOI. in Japan $[2,3]$. In this method, an injected beam is kicked by the PMM off-axis and captured into the ring acceptance by damping while the stored beam passes through the center of the PMM where the magnetic field is almost zero. This method allows us to inject an electron beam into a storage ring without a large perturbation on the stored beam during the top-up operation. However, the injection efficiencies could be poor for this PMM injection scheme particularly when the injected beam size is so large that the beam will receive a large nonlinear kick from PMM. Additionally, the stored beam will receive a gradient kick at the center due to multipole field components, which will increase the beam size.

To overcome this problem, BESSY adapted the PMM injection idea and came up a novel nonlinear kicker (NLK) design, which has a nonlinear magnetic field distribution with a maximum value off-axis where the injected beam is kicked and zero or near-zero dipole and quadrupole fields at the stored beam center [4]. Thus, the injected beam receives a maximum kick from the NLK and the stored beam will receive much less perturbations. This nonlinear magnetic field distribution can be created by eight conductors symmetrically located in four quadrants of a transverse space. This nonlinear kicker magnet has been designed as a short in-vacuum magnet and tested in the BESSY II storage ring. During the injection process the on-axis stored beam remains undisturbed, whereas the offaxis injected beam is deflected by the maximum field 
and accumulated finally by radiation damping. Due to its transparent injection, compactness and simplicity, several facilities have adopt BESSY's NLK design. This includes MAX-IV [5], Sirius [6], Diamond [7], SLS-2 [8] light sources.

At the Advanced Light Source (ALS) of Lawrence Berkeley National Laboratory, we are now engaged in the construction of a new hard x-ray beam line and insertion device for protein crystallography [9]. The scope of work entails the reconfiguration of ALS Sectors $1-3$ to make room for the new insertion device. To consolidate the space, the NLK injection becomes attractive to this project. This technology will, in principal, ultimately allow us to remove the conventional bump injection magnets presently located in Sector 1 and to facilitate top-off injection without noticeable perturbations on the stored beam.

The NLK injection also remains attractive to Advanced Light Source Upgrade (ALS-U) which is an ongoing upgrade project and will provide $\mathrm{x}$-ray beams at least 100 times brighter than those of the existing ALS [10]. One enabling technology for this upgrade is on-axis swap-out injection with fast kicker magnets to exchange bunch trains between the storage ring and a accumulator ring. The accumulator is a full-energy synchrotron and installed in the same storage ring tunnel. It has a similar dynamic performance as the current ALS storage ring, allowing for off-axis injection. The NLK injection has become a candidate method to inject beams from the booster to the accumulator.

In Sec. II, we discuss the working principle of NLK injection and its design consideration. In Secs. III and IV, we present the NLK injection optimizations for both ALS and ALS-U to achieve maximum injection efficiencies. We conclude the paper in Sec. V.

\section{THEORY OF NLK INJECTION}

\section{A. Magnetic field distribution}

To a create nonlinear distribution of magnetic fields with a maximum value off axis and a zero or near-zero field at the center, the nonlinear kicker can be constructed as 8 current-driven conductors with a horizontal and vertical mirror symmetry as shown in Fig. 1. There are two conductors on each transverse quadrant carrying current in opposite directions. The inner four conductors carry the current in the same direction, and the outer four conductors carry the current with an opposite polarity. The symmetry about the horizontal mid-plane enforces a zero horizontal magnetic field on this plane, whereas the symmetry about the vertical plane leads to a zero vertical dipole field at the geometry center of the kicker.

The magnetic fields of this NLK can be estimated using Ampere's Law assuming infinitely long straight conductors. This estimation is especially good for a long kicker with a small transverse size. More accurate magnetic fields can be calculated using the Biot-Savart Law

$$
d B=\frac{\mu_{0}}{4 \pi} \frac{I d \vec{l} \times \hat{r}}{r^{2}}
$$

where $\mu_{o}$ is the permeability of free space, $d \vec{l}$ is the infinitesimal length of conductor, $r$ and $\hat{r}$ are the radial distance and direction of observation point, respectively. Integrating this equation along the conductors path will give the magnetic fields of the NLK.

Figure 1 shows the vertical magnetic field profile in the horizontal mid-plane. It can be seen that the magnetic field of this NLK has a peak value off-axis, and near-zero dipole and quadrupole fields at the center. Since the inner and

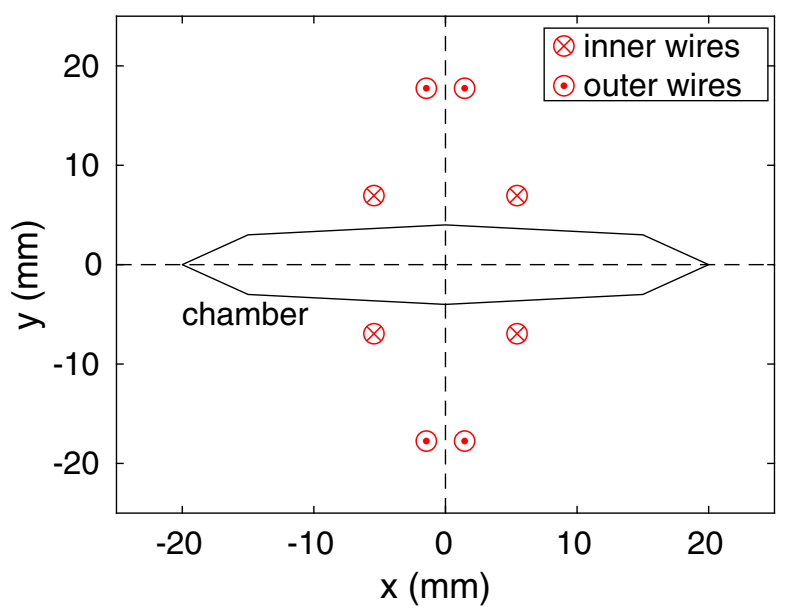

(a)

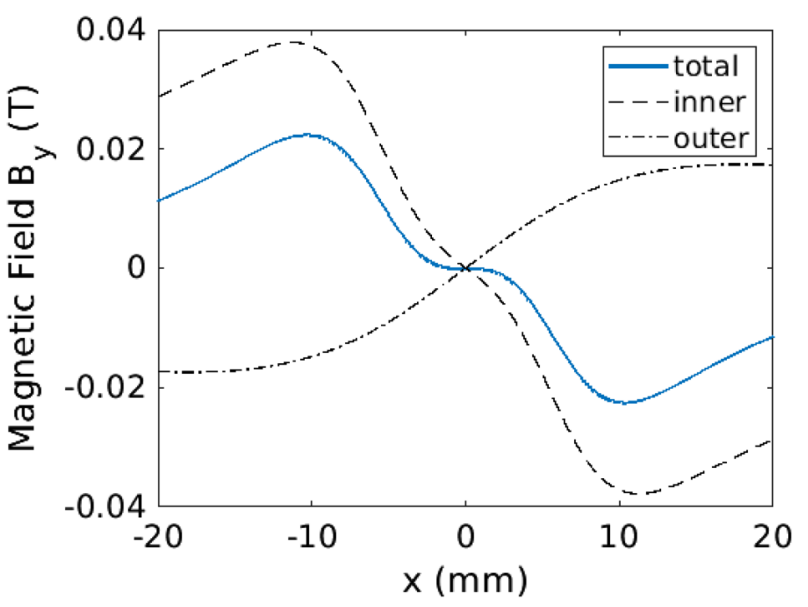

(b)

FIG. 1. The conductors geometry and the vertical magnetic field profile for an optimized ALS nonlinear kicker. (a) 8 currentdriven conductors symmetrically located in four quadrants of a transverse plane. The inner and outer four conductors carry current in an opposite direction. (b) The vertical magnetic field is observed along the horizontal mid-plane. The blue solid line represents the total field contribution from 8 conductors, the dashed line represent the field contributions from the inner four conductors and the dash-dot line from the outer four conductors. 
outer conductor locations are independent of each other, they can be adjusted to shape the field profile at the peak to improve the injection efficiency, and to minimize the magnetic fields at the center region to reduce the perturbation on the stored beam. The magnetic fields independently generated by the inner four conductors and outer four conductors are shown in Fig. 1. It can be seen that these two field components have a different directions due to the opposite polarity of the current, which could help shape the field profile around peak and center regions. In particular, it allows us to achieve a near zero quadrupole field at the center region by optimizing the conductor positions.

Certainly, a nonlinear kicker can be constructed with more conductors and/or a breaking symmetry to generate arbitrary magnetic field distribution [8]. However, the symmetric 8 conductors design is the simplest and the most effective one to achieve a good injection efficiency.

\section{B. Effect on injected beams}

In order to have a better injection efficiency, the injected beam usually arrives at the peak field location of the NLK. After the kick, the injected beam transverse momentum is reduced, therefore its oscillation amplitude is decreased in the following turns. If the oscillation amplitude is smaller than the ring acceptance, the injected beam will eventually be captured by the ring through synchrotron radiation damping. Figure 2(a) illustrates the NLK injection with a single particle tracking through the NLK and ALS-U accumulator ring for the first turn. The ALS-U accumulator ring is a Triple-Bend Achromat lattice with 12 superperoids, similar to the current ALS lattice. The beam is injected with an offset of about $16 \mathrm{~mm}$ at the injection point (IP) in the first straight section right after the thin septum. The NLK is located at the center of the second straight section of the ring. When the NLK is turned off, the injected beam has a large oscillation amplitude beyond the ring acceptance and will be lost. With the NLK turned on, the oscillation amplitude is reduced to the level of within the ring acceptance and will eventually be damped down by the radiation damping. Figure 2(b) illustrates the NLK injection in a normalized space. When the injected beam passes through the kicker, it receives a momentum kick $\Delta P$, its oscillation invariant $\varepsilon_{0}$ will be reduced to a smaller value $\varepsilon$. When the reduced invariant $\varepsilon$ is less than the ring acceptance, the injected beam will be captured by the ring.

Since the NLK has a nonlinear distribution of magnetic fields around the peak region, the injected beam will receive a nonlinear kick. For a small injected beam, this nonlinear kick effect could be small and would not affect inject efficiency [11]. However, for a large injected beam, such as the beam injected from the ALS booster, it could have a significant impact on the injection efficiency. The ideal kicker design for the NLK injection is to have a flattop magnetic field distribution at the peak region. However, this is difficult to achieve with the symmetrical 8 conductors design. In the Sec. III, we will discuss how to optimize the conductor positions to shape the magnetic field profile to improve the injection efficiency.

\section{Impact on stored beams}

One of the attractive features of the NLK injection is the perturbations reduction on the stored beam because of zero or near-zero magnetic fields at the stored beam center. However, this zero or near-zero fields are not guaranteed by any design choices. It is important to specify the field tolerance requirement at the center field-free region for the kicker design. This center field-free region can be defined as a several sigma of the stored beam size. The two dominating field components in this region are dipole and quadrupole fields.

The dipole field could cause the beam centroid to oscillate. The oscillation amplitude is given by $\beta \theta$, where $\beta$ is the beta function at the NLK and $\theta$ is the kick angle.

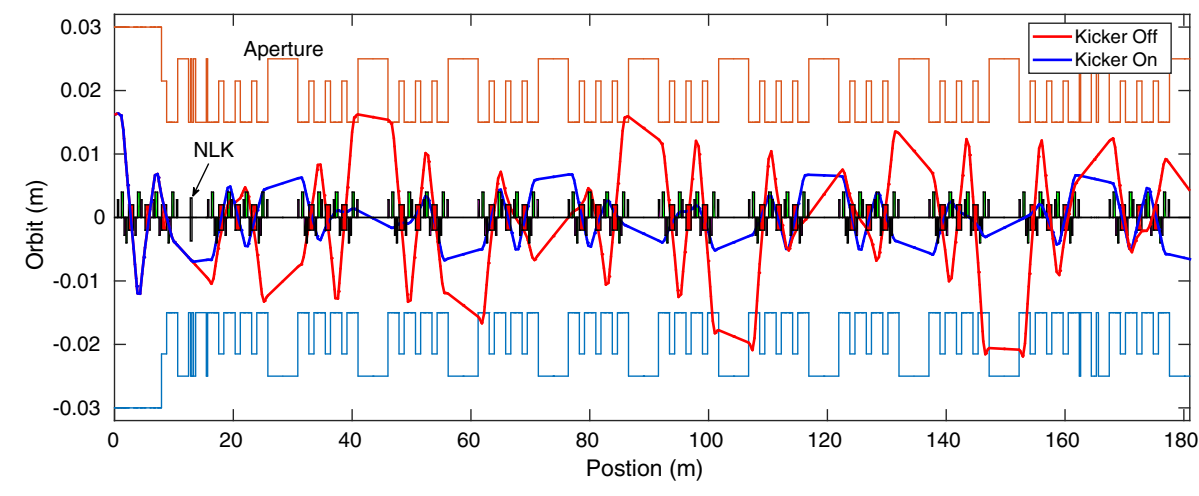

(a)

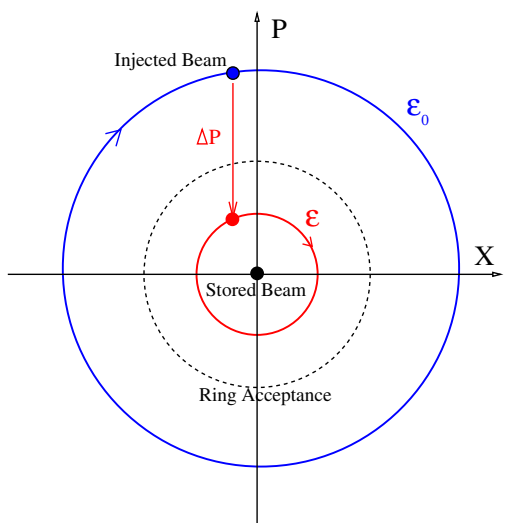

(b)

FIG. 2. Illustration of a nonlinear kicker injection into the ALS-U accumulator ring. (a) A single particle tracking for the first turn with the NLK kicker turned ON and OFF. (b) Diagram of the NLK injection in a normalized phase space. The large blue, median dash, and small red circles represent the injection invariant, ring acceptance and reduced invariant of the injected beam, respectively. 
To make injection as transparent as possible, the oscillation amplitude should be less than $\% 5$ of the beam size (10\% for a relax requirement), which is usually specified by beam users experiments.

The quadrupole field in the fieldfree region will not cause the beam centroid to oscillate, but it could increase the beam size. The beam size increasement can be estimated by [12]

$$
\frac{\Delta \sigma}{\sigma}=\frac{B^{\prime}}{2 B \rho} L \beta_{x}+0.4\left(\frac{B^{\prime}}{2 B \rho} L \beta\right)^{2},
$$

where $B \rho$ is the magnet rigidity, $B^{\prime}$ is the quadrupole gradient in the fieldfree region, $L$ is the kicker length and $\beta$ is the beta function of the stored beam at the kicker location. To have a transparent injection, the relative beam size increase caused by the quadrupole field should be less than $5 \%$ or $10 \%$.

These field tolerance requirements at the center fieldfree region could be major design constraints, limiting the design choices of NLK. In the later sections, we will discuss the impact of these tolerance requirements on both ALS and ALS-U NLK designs.

\section{Injection tracking and optimization}

Injection with a NLK can be studied using the particle tracking technique. As mentioned before, the magnetic fields of a NLK can be calculated by the Ampere's law or the Biot-Savart law. With calculated fields, particles can be tracked through the NLK using the leapfrog drift-kick-drift technique over many thin segments of the NLK. More accurately, the differential equations of a particle's motion influenced by magnetic fields should be integrated through the NLK using the Runge-Kutta methods [13]. However, the previous method can be easily implemented and could be accurate enough if using a large numbers of segments.

The injection efficiency with a NLK can be affected by many factors, such as the injected beam angle and offset, NLK magnetic field distribution, injected beam Twiss parameters and so on. A small beam offset and angle at the NLK location are preferred for a higher injection efficiency. A small offset is a necessary requirement for a small injection invariant as illustrated in Fig. 2 therefore a higher injection efficiency. A small beam angle requires a small kick angle to compensate. These beam offset and angle at the NLK location can be affected by the initial injection condition (i.e., the injected beam angle and offset at the injection point (IP) right after the septum) and the location of the NLK. When we design a transfer line and septum, this initial injection condition needs to be minimized. We can also optimize the location of NLK to reduce the beam offset and angle at the NLK location. To reduce the offset, the NLK should be placed downstream as far as possible from the injection point (IP) if both NLK and IP are located in the same straight section. To reduce the angle, the NLK can be placed in the downstream straight section of the IP to generate betatron oscillation through the arc section. In general, to achieve a higher injection efficiency, we need to optimize both the initial injection condition and the location of NLK.

The injection efficiency can be affected by the magnetic field distribution of the NLK, i.e., the peak field location and profile. In general, to have a better efficiency, the peak field should be at the arriving position of the injected beam and the peak profile should have a flat-top distribution. The field distribution is given by the conductor positions as we discussed before. Therefore, to have a better injection efficiency, we need to optimize the conductor positions of the NLK.

The injection efficiency can also be affected by the Twiss parameters of injected beam. Without matching the injected beam Twiss parameters to the stored beam Twiss parameters, a small initial oscillation amplitude of the injected beam could result in a large ring acceptance requirement. Therefore, to achieve a better injection efficiency, the injected beam Twiss parameters need to be optimized.

All the parameters mentioned above can be used as tuning knobs to optimize the NLK injection. However, these tuning knobs are not completely independent of each other. Sometimes, they can even compete with each other. For example, the injected beam offset at the injection point cannot be very small because it is determined by the septum sheet location and the separation between the injected beam and septum sheet. If the injected beam offset is too small, the septum sheet has to be closer to the stored beam, which will reduce the dynamic aperture of the ring and therefore the injection efficiency. There are optimal settings for these tuning knobs. Optimization of these knobs is a multivariable and multiobjective optimization problem. Multiobjective genetic algorithm (MOGA) [14] is well suitable for this problem. This algorithm has been widely used in the accelerator community to optimize lattices [15]. Here we will use this algorithm to optimize the NLK injection. The optimization of NLK injection is often constrained by design and tolerance requirements. For example, the minimal heights of inner conductors are limited by the vertical vacuum chamber size, and the magnetic fields in the fieldfree region should meet the tolerance requirements to minimize perturbations on the stored beam. The NLK optimization with MOGA is a time consuming process because the injection efficiency is evaluated by multiparticle tracking technique. To decrease the turnaround time of the optimization process, a parallel computing technique with clusters can be applied to optimize NLK injections.

In the following sections, we will discuss NLK injection optimizations for both ALS and ALS-U facilities.

\section{OPTIMIZATION OF NLK INJECTION FOR ALS}

The Advanced Light Source (ALS) is now engaged in the construction of a new hard $\mathrm{x}$-ray beam line and insertion 


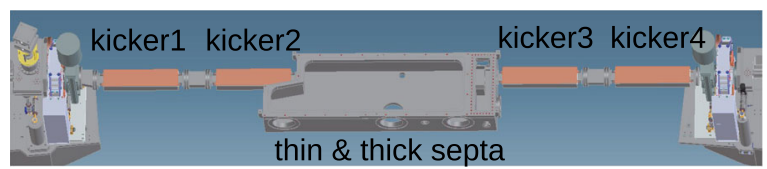

Sector 1

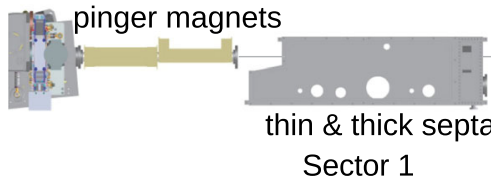

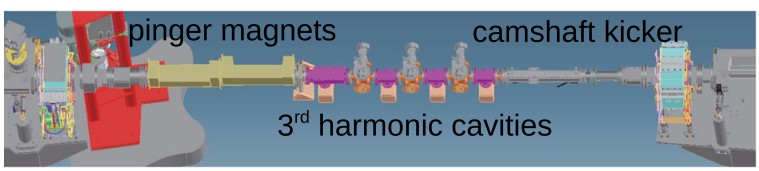

Sector 2

(a)

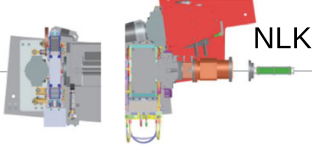

(b)

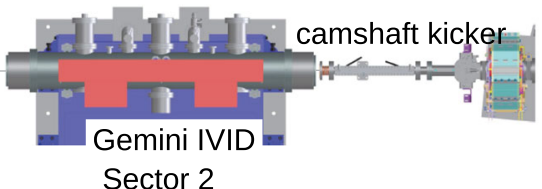

Sector 2

FIG. 3. Layout of sector 1 and sector 2 straights before (a) and after (b) the melioration of the ALS injection system.

device for protein crystallography [9]. The scope of work entails the reconfiguration of ALS sectors 1-3, where the injection, cavities, pinger magnets and feedback kickers are located, to make room for the new insertion device (Gemini IVID) installed at sector 2 . The project will require the melioration of the ALS injection system. A key aspect of this work is the development and integration of a nonlinear injection kicker (NLK) magnet at sector 2 to facilitate the top-off injection without noticeable perturbations on the stored beam. Due to the compactness of NLK, this injection scheme will allow us to remove the four bump injection kicker magnets presently located in ALS Sector 1 to provide space for other devices. Figure 3 shows the layout of sector 1 and sector 2 straights before and after the melioration of the ALS injection system. The 3rd harmonic cavities in the sector 2 straight will be relocated to the sector 3 straight which is not shown in the figure.

The NLK design for ALS evolved from the original concept developed at BESSY [4]. This concept positions eight conductors at $45 \mathrm{deg}$ from the axis. The inner conductors are driven with current in one direction and the outer conductors with current in the opposite direction. The original BESSY design has been modified at ALS by scaling the vertical aperture and conductor current. The vertical magnetic field profile of this modified NLK is shown in Fig. 4. Tracking study shows that only about $70 \%$ injection efficiency can be achieved with this modified NLK, which is limited by the shape and location of the peak field profile of this kicker. To effectively inject a large beam (about $2.5 \mathrm{~mm}$ in RMS) provided by the existing ALS booster which has a natural emittance of about $274 \mathrm{~nm}$-rad, it becomes essential to optimize the NLK peak field profile and location. In particular, a profile that has a wider zero field region near the axis, rises more steeply to the peak, and then decays more slowly would improve the injection efficiency [16]. This field profile can be shaped by adjusting the positions of the 8 conductors symmetrically around the NLK center.

Multiobjective genetic algorithm (MOGA) [14] has been used to optimize the NLK injection for ALS. To shape the magnetic field profile, there are 4 parameters available for conductor positions ( 2 for inner conductors and 2 for outer conductors) and 1 parameter for the conductor current. The horizontal Twiss parameters ( $\beta_{x}$ and $\alpha_{x}$ functions) of the injected beam, as well as the injected beam offset (x) and angle (xp) at the injection point also need to be tuned to achieve a better injection efficiency. The vertical Twiss parameters are not tuned during the injection optimization because the beam is injected in the horizontal plane and the ALS storage ring has a relative large vertical acceptance. The location of the thin septum sheet (xseptum) is fixed at $15 \mathrm{~mm}$ by the current off-axis injection layout. In total, there are 9 parameters available to optimize the NLK injection. The optimization objectives are to maximize the injection efficiency and minimize the conductor current.

The optimization is constrained by the tolerance requirement of the dipole and quadrupole fields at the fieldfree region. To make the injection as transparent as possible for beam users, the perturbations on the stored beam should be less than $5 \%$ of the stored beam size. Figure 5 shows perturbations on the stored beam calculated by both tracking and analytical methods for different dipole and

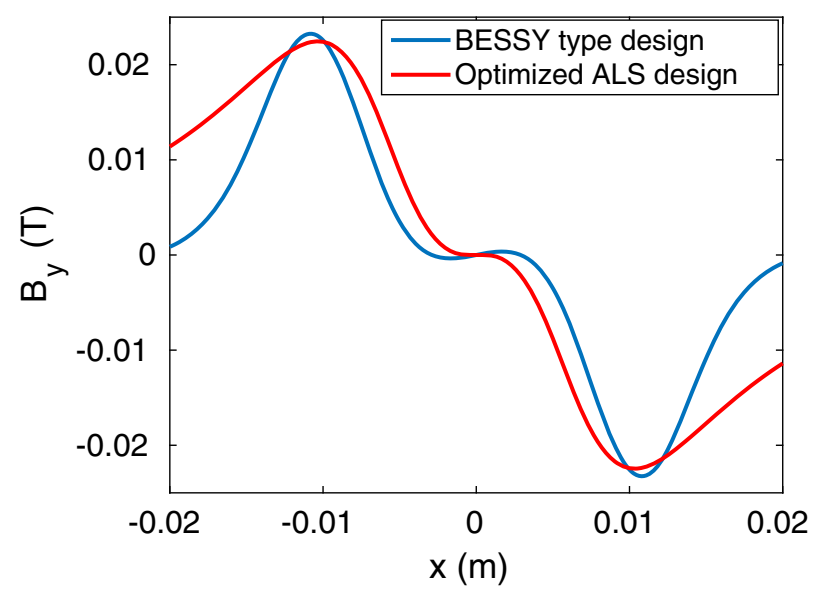

FIG. 4. Vertical magnetic field profiles of NLKs for the BESSY type design and an optimized ALS design. 


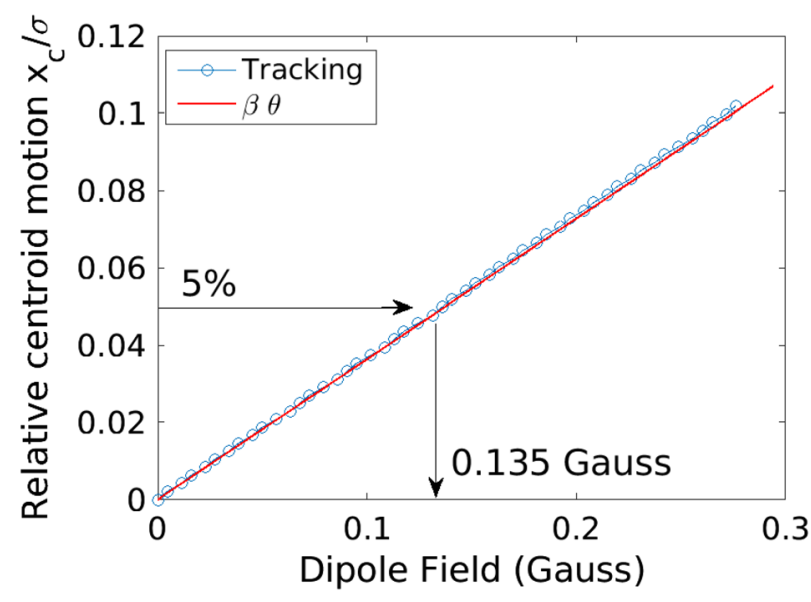

(a)

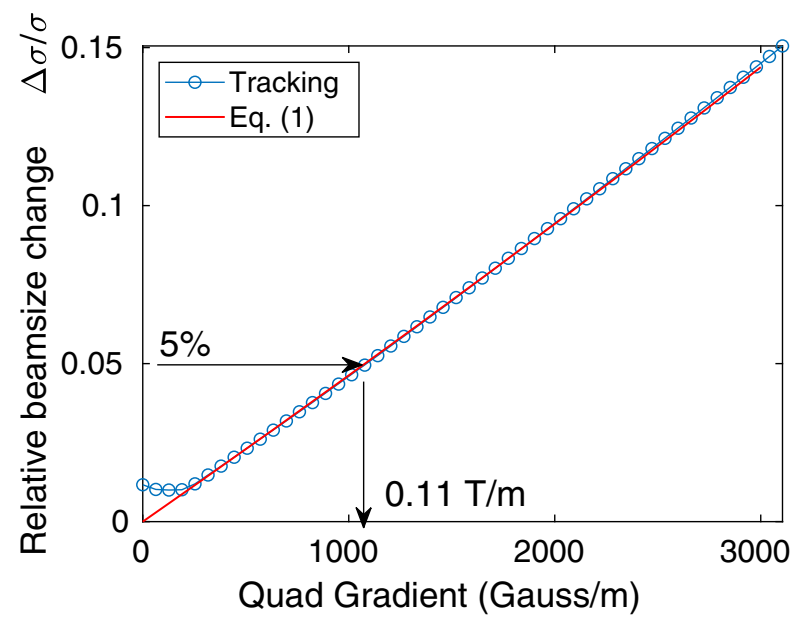

(b)

FIG. 5. Perturbations on the stored beam for different dipole (a) quadrupole (b) field strengths at the fieldfree region of NLK.

quadrupole field strengths at the field-free region of the NLK. For the tracking study, 1000 particles are tracked through the ALS storage ring for 10000 turns and the maximum beam-centorid oscillation amplitude and beam size are calculated for each dipole and quad strength. The tracking results agree with analytical calculation very well. $5 \%$ of the beam size perturbation requires that the dipole field at the field-free region should be less than 0.135 Gauss and the quadrupole field gradient should be less than $0.11 \mathrm{~T} / \mathrm{m}$ as shown in Fig. 5. The fieldfree region is defined as $\pm 0.4 \mathrm{~mm}$ around the stored beam center, which is about \pm 2 sigma of the stored beam size. The optimization is also constrained by the minimal height of inner conductor positions which is given by the vertical chamber size. The conductor positions should be higher than $7 \mathrm{~mm}$ in order to have an $11 \mathrm{~mm}$ full vertical chamber size, which will give the minimal $1.5 \mathrm{~mm}$ thickness required for chamber wall and conductor radius. A very narrow chamber will place challenges on its mechanical design. The shimmability is another import design consideration for the NLK. The 8 conductors will be embedded in the grooves of the ceramic chamber. Any positioning error of these conductors will introduce field errors at the fieldfree region. These errors should be corrected after constructing the kicker. Our study shows that the dipole field errors can be corrected by shimming the top two conductors, which requires the change of the dipole field larger than 3 Gauss per $400 \mu \mathrm{m}$ horizontal offset of the top two conductors. This shimming constraints is implemented in the injection optimization.

To evaluate the injection efficiency, 5000 particles were tracked through the septum channel, the nonlinear kicker and then the storage ring lattice for 1000 turns. At the end of 1000 turns, the particle surviving rate, i.e., the injection efficiency, was calculated. For this tracking study, $3 \times 10^{-4}$ relative gradient errors and $5 \times 10^{-4}$ skew quadrupole errors are introduced into the storage ring lattice, which generate the same level of beta-beating and coupling as we measured using the LOCO technique. The solutions at different generations optimized by MOGA are shown in Fig. 6. After the 200th generation the solutions are well converged to a Pareto front. As it can be seen from the Pareto front, the optimal injection efficiency that we can achieve is about $91 \%$ with the current of 769 A. The parameter settings for the solution marked by a red circle in the plot are listed in Table I. The magnetic field profile for this optimized NLK design is shown in Fig. 4. Compared to the BESSY's design, the optimized ALS design has more a flatter field top and the peak is closer to the center. For this solution, the required $\beta_{x}$ and $\alpha_{x}$ function are $38.27 \mathrm{~m}$ and -0.0865 , respectively; and the inject beam offset (x) and angle (xp) are $-20.849 \mathrm{~mm}$ and $80.088 \mu \mathrm{rad}$, respectively. With this solution, the injection errors have been studied using tracking techniques. The results are summarized in Table II. It shows that about several percent of the injection efficiency variation from $84 \%$ to $93 \%$ has been observed even for a very large error setting. This illustrates that our

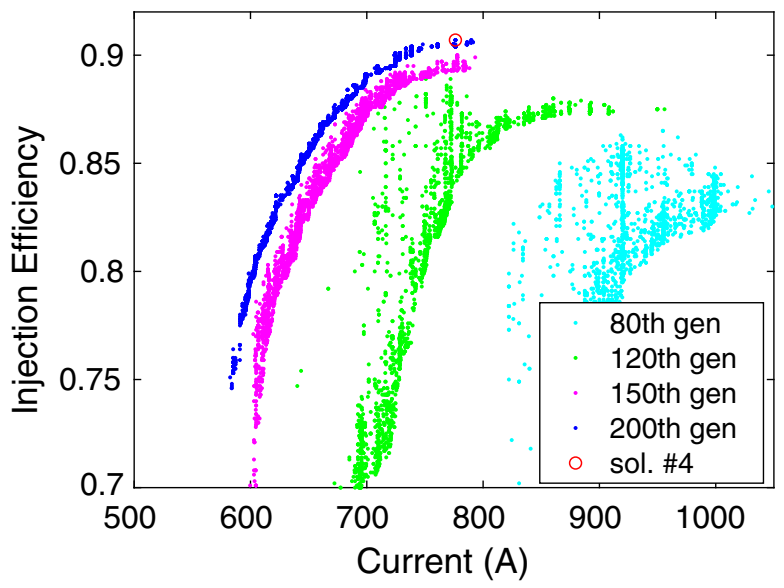

FIG. 6. Pareto front from the ALS NLK injection optimization using MOGA. 
TABLE I. Parameters of optimized NLK injections for both ALS and ALS-U.

\begin{tabular}{|c|c|c|c|c|c|c|c|c|c|c|c|}
\hline & I (A) & $\mathrm{X}_{\mathrm{in}}(\mathrm{mm})$ & $\mathrm{Y}_{\text {in }}(\mathrm{mm})$ & $\mathrm{X}_{\text {out }}(\mathrm{mm})$ & $\mathrm{Y}_{\text {out }}(\mathrm{mm})$ & $\alpha_{x}$ & $\beta_{x}(\mathrm{~m})$ & $\begin{array}{c}\text { Injection } \\
\text { offset (mm) }\end{array}$ & $\begin{array}{c}\text { Injection } \\
\text { angle (mrad) }\end{array}$ & $\begin{array}{c}\text { Septum } \\
\text { offset }(\mathrm{mm})\end{array}$ & $\begin{array}{c}\text { NLK } \\
\text { offset (mm) }\end{array}$ \\
\hline ALS & 768.89 & 5.479 & 7.043 & 1.883 & 17.603 & -0.086 & 38.27 & 20.85 & 0.080 & 15.00 & 0 \\
\hline ALS-U & 852.69 & 1.220 & 8.688 & 5.372 & 18.417 & -0.445 & 13.364 & 16.17 & 0.414 & 10.101 & 0.472 \\
\hline
\end{tabular}

NLK injection is robust to these injection error effects. The injection efficiency limited to about $90 \%$ is mainly due to the large injected beam size from the ALS booster. It is also limited by the tolerance and vertical chamber size requirements, which do not allow us to move the peak field closer to the stored beam center. However, it is still a big improvement over the current local bump injection scheme with which only about $50 \%$ injection efficiency is achieved. For this scheme, the injection efficiency is limited mainly due to a small horizontal phase advance of the storage ring and a large residual local bump amplitude so that the injected particles are lost at the septum sheet at the second turn.

As shown in Fig. 4, the dipole and quadrupole field components in the fieldfree region are small compared to

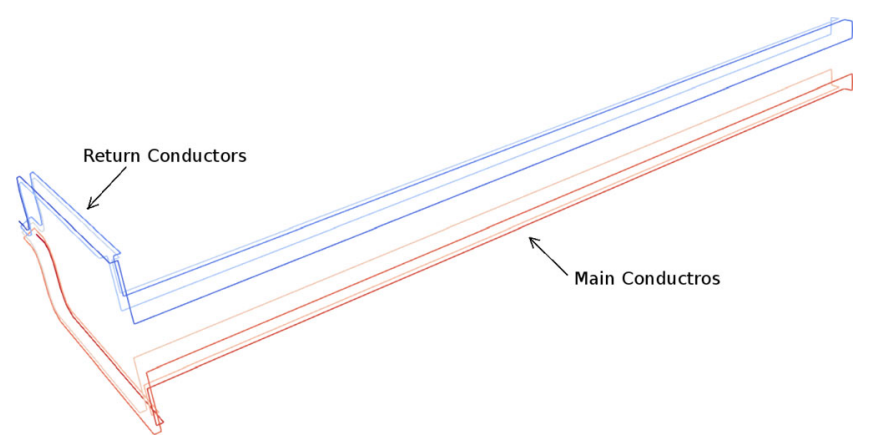

(a)

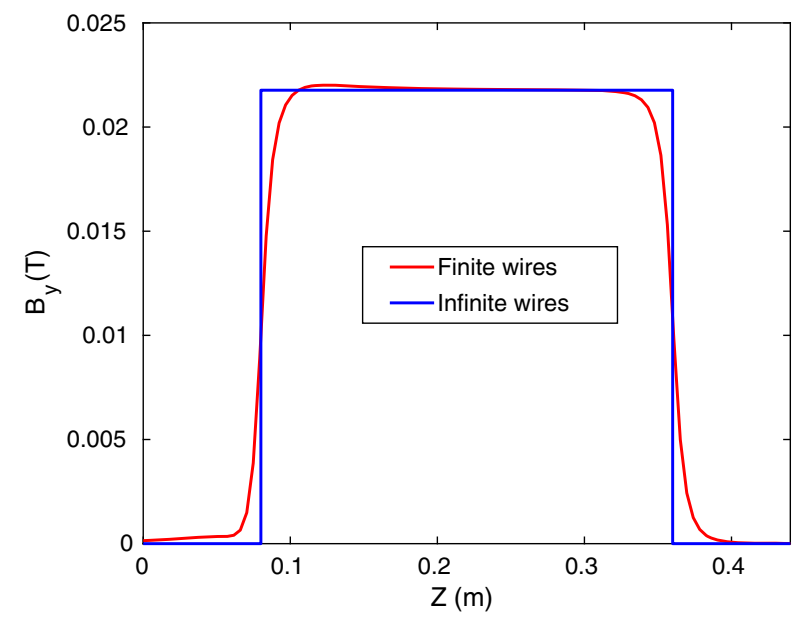

(b)

FIG. 7. Finite conductors model (a) and its vertical magnetic field (b) compared to the infinite long conductor model. the BESSY design, attributing to the field tolerance constraints which is imposed during the MOGA optimization. In the field free region $( \pm 0.4 \mathrm{~mm})$, the maximum dipole field and quadrupole field gradient are all within the tolerance requirements of 0.135 Gauss and $0.11 \mathrm{~T} / \mathrm{m}$, respectively.

To speed up the optimization process, an infinite long conductor model has been used in the MOGA runs. To validate this assumption, a $3 \mathrm{D}$ finite conductor model with return loops is also implemented in the tracking code with the Biot-Savart law and Runge-Kutta integrator as discussed in Sec. II. Figure 7 shows the 3D conductor model and its magnetic field compared to the infinite long conductor model. The finite conductor model introduces fringe field effects. But tracking results show that this effect is small and does not have a significant impact on the NLK injection efficiency.

At ALS, we are developing a prototype of the NLK based on the solution optimized above [16]. The CAD model of this kicker is shown in Fig. 8. It has a magnetic length of $28 \mathrm{~cm}$ and the aperture size is $32 \mathrm{~mm} \times 11 \mathrm{~mm}$ (width $\times$ height). The chamber is ceramic with about $8 \mu \mathrm{m}$ titanium coating and provides the structural support and positioning of the conductors. The impacts of this coating on the heat load and magnetic field due to eddy current effects have been investigated in paper [16], This coating will result in the field attenuation by approximately $1.1 \%$ at the peaks and also dissipate heat approximately $10 \mathrm{~W}$. With the addition of a $10 \mathrm{~m}$ cable between the power supply and the wiring interconnects at the magnet, the voltage required from the power supply is $5 \mathrm{kV}$. The mechanical and electrical designs of this NLK have been completed. Right now we are facing some technical challenges of the ceramic chamber design.

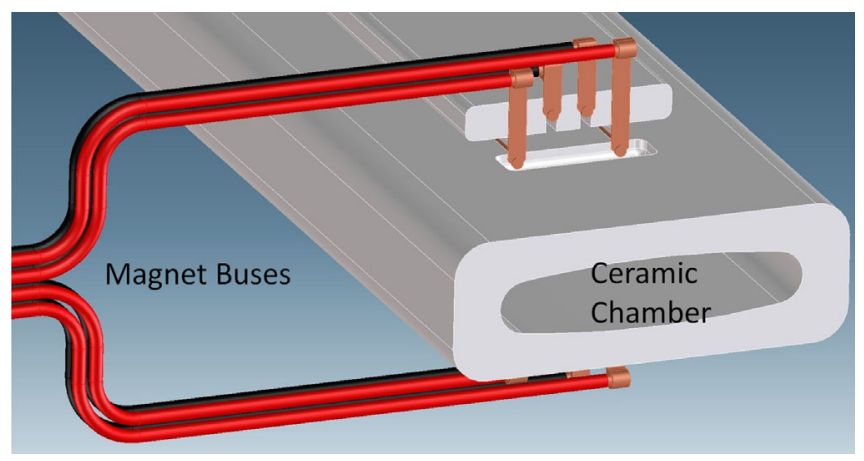

FIG. 8. The prototype of the ALS NLK magnet. 
TABLE II. Injection efficiency affected by the injection errors.

\begin{tabular}{lcccccccccccc}
\hline \hline & \multicolumn{3}{c}{ Septum location, xseptum $=-15 \mathrm{~mm}$} & \multicolumn{3}{c}{ Injection offset, $\mathrm{x}=-20.849 \mathrm{~mm}$} & \multicolumn{3}{c}{ Injection angle, xp $=80.088 \mu \mathrm{m}$} \\
\hline Error & $+1 \mathrm{~mm}$ & $+0.5 \mathrm{~mm}$ & $-0.5 \mathrm{~mm}$ & $-1 \mathrm{~mm}$ & $+1 \mathrm{~mm}$ & $+0.5 \mathrm{~mm}$ & $-0.5 \mathrm{~mm}$ & $-1 \mathrm{~mm}$ & $+80 \mu \mathrm{m}$ & $+40 \mu \mathrm{m}$ & $-40 \mu \mathrm{m}$ & $-80 \mu \mathrm{m}$ \\
Eff $(\%)$ & 92.6 & 91.8 & 89.7 & 88.3 & 88.0 & 89.7 & 91.3 & 91.2 & 84.2 & 88.8 & 88.7 & 83.4 \\
\hline \hline & & & & & & $\alpha_{x}=-0.0865$ & & & $\varepsilon_{0}=274 \mathrm{~nm}$ & \\
\hline Error & $+10 \mathrm{~mm}$ & $+5 \mathrm{~mm}$ & $-5 \mathrm{~mm}$ & $-10 \mathrm{~mm}$ & +0.08 & +0.04 & -0.04 & -0.08 & $+30 \mathrm{~nm}$ & $+15 \mathrm{~nm}$ & $-15 \mathrm{~nm}$ & $-30 \mathrm{~nm}$ \\
Eff $(\%)$ & 89.5 & 90.2 & 90.9 & 90.9 & 90.6 & 90.9 & 90.8 & 90.7 & 88.3 & 89.5 & 91.7 & 92.5 \\
\hline \hline
\end{tabular}

\section{OPTIMIZATION OF NLK INJECTION FOR ALS-U}

Advanced Light Source Upgrade (ALS-U) is an ongoing upgrade project at Lawrence Berkeley National Lab which will provide $\mathrm{x}$-ray beams at least 100 times brighter than those of the existing ALS [10]. The upgraded ALS will occupy the same facility as the current ALS, replacing the Triple Bend Achromat storage ring lattice with a compact multibend achromat lattice which has a very small natural emittance of about $100 \mathrm{~nm}$-rad. One of the consequences of producing such a small emittance is a small ring dynamic aperture into which an electron beam cannot be injected using a conventional off-axis injection scheme. To overcome this challenge, ALS-U will apply on-axis swap-out injection to exchange bunch trains between the storage ring and a full-energy accumulator ring. This accumulator will be installed in the storage ring tunnel and will also act as a damping ring for the booster beam. It has a similar dynamic aperture as the current ALS storage ring and allows for offaxis injection from the existing booster. Different injection methods have been investigated to inject beams from the booster to the accumulator and the NLK injection is one of the candidate injection methods [17].

Although avoiding perturbations to the stored beam in the accumulator is not a major concern because this beam is not used for user experiments, an NLK injection still remains attractive for its compactness and simplicity. Recognizing the relaxed tolerance requirement of magnetic fields in the center field-free region is important to determine the most effective NLK design for the accumulator. In particular, it will allow us to increase the NLK vertical chamber aperture size without sacrificing the injection efficiency, therefore easing the mechanical design of NLK. It also gives us an additional tuning knob, the horizontal offset of the NLK, enabling us to bring the peak field even closer to the stored beam center. Guiding by this principle, we carry out the NLK injection optimization with MOGA following a similar approach used for the ALS NLK injection optimization.

In addition to the 9 tuning knobs used in the ALS NLK optimization, the thin septum sheet offset and the horizontal offset of the NLK are two additional tuning knobs. Compared to the ALS injection, the tolerance requirements of dipole and quadrupole fields in the field-free region are much more relaxed: the dipole field is less than 100 Gauss corresponding to $0.42 \mathrm{mrad}$ kick to the stored beam; the quadrupole field gradient is less than $20 \mathrm{~T} / \mathrm{m}$ which could increase the stored beam size by about a factor of 12 . These magnet field strength are well within our tolerance requirements of keeping the stored beam inside the accumulator ring during the injection. Another constraint is that the vertical positions of the inner conductors are higher than $8.5 \mathrm{~mm}$ for a large vertical chamber size consideration. The accumulator ring lattice after LOCO corrections is used in this optimization. To reduce the kick strength requirement, the NLK kicker is installed at the center of the 2nd straight section.

Figure 9 shows a Pareto front obtained from a MOGA run after a few hundred generations. About 99\% injection efficiency can be achieved with the solution marked by a red circle in the plot. The conductor current for this solution is about $850 \mathrm{~A}$. The parameters for this solution are listed in Table I. Figure 10 shows conductors position and magnet field profile. The vertical position of inner conductors is at $8.688 \mathrm{~mm}$ which meets our vacuum aperture size requirement. Compared to the magnetic field profile of the ALS NLK as shown in Fig. 4, the ALS-U NLK does not have a flat field distribution in the center fieldfree region mainly

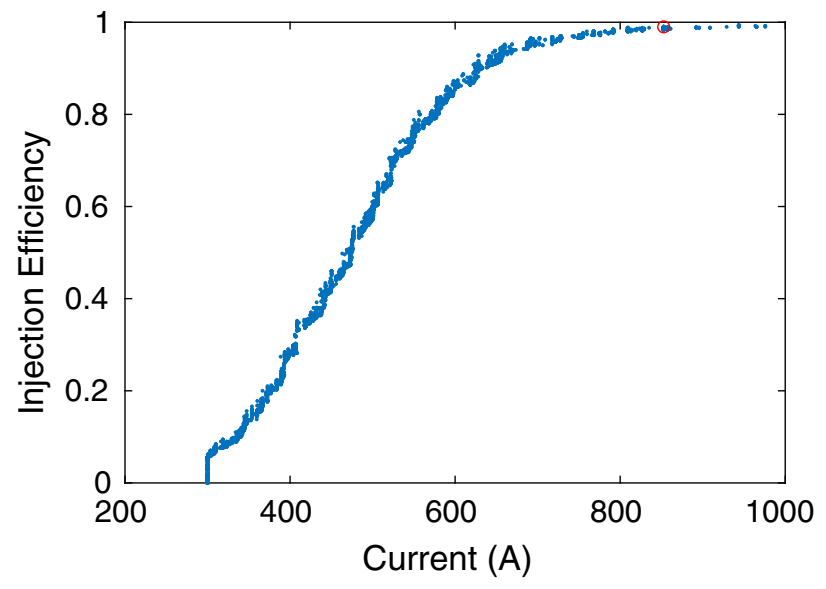

FIG. 9. A Pareto front from the ALS-U NLK injection optimization using MOGA. The $99 \%$ injection efficiency could be achieved with the solution marked by a red circle. 


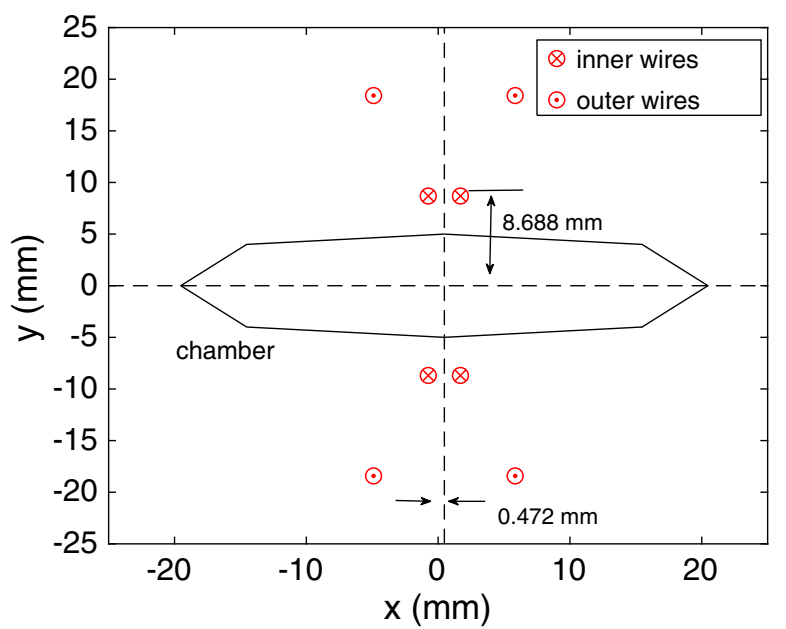

(a)

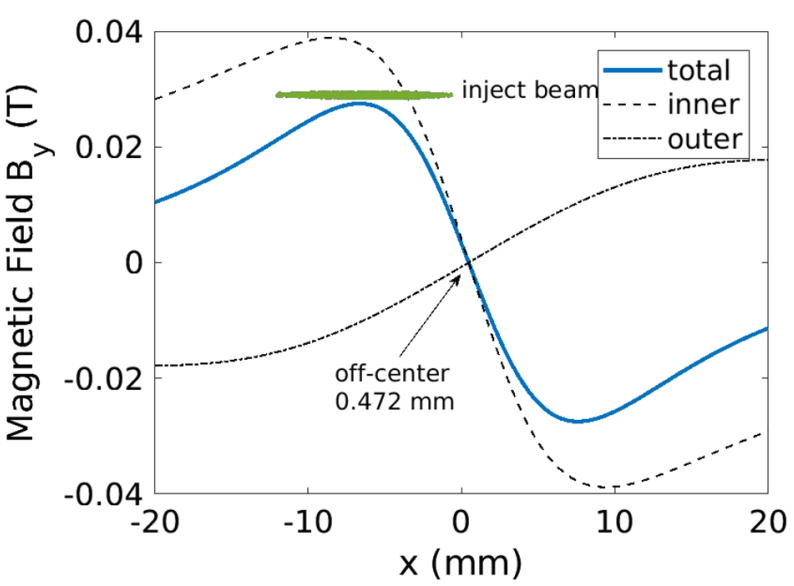

(b)

FIG. 10. The NLK conductor geometry for ALS-U (a) and its vertical magnetic field distribution (b). The vertical position of inner conductors is at $0.8688 \mathrm{~mm}$ and the NLK is horizontally offset by $0.472 \mathrm{~m}$. The inject beam distribution at the NLK is plotted on the top of the field profile. The centroid of the beam is at $6.8 \mathrm{~mm}$.

due to the relaxed tolerance requirements. This relaxation allows us to bring the peak field closer to the stored beam center even with a large vacuum chamber. In addition, the NLK is horizontally offset by about $0.472 \mathrm{~mm}$ to further move the peak field closer to the center. This solution requires the septum sheet located at $10.101 \mathrm{~mm}$ offset in order to bring the injected beam closer to the center; the separation between the injected beam and the septum sheet is about $5.075 \mathrm{~mm}$, about 2.5 sigma of the injected beam size. The inject beam offset and angle at the injection point are $16.176 \mathrm{~mm}$ and $0.414 \mathrm{mrad}$, respectively. The beam distribution right before the NLK is also shown in Fig. 10(b). The beam centroid is at about $6.843 \mathrm{~mm}$, which is right at the location of the NLK peak field.

Multiparticle tracking is carried out to study injection, damping and capturing process with the solution optimized

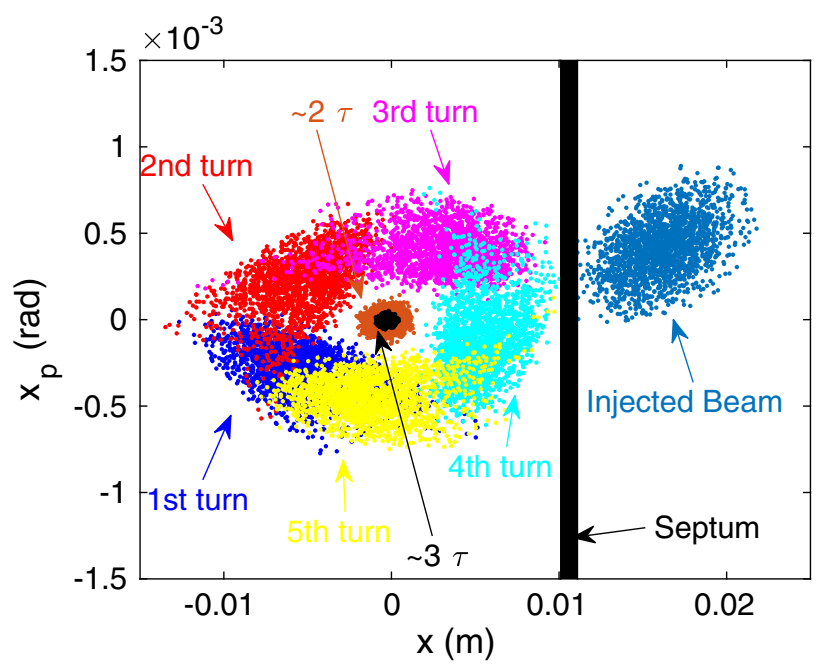

FIG. 11. Beam distribution for the first few turns and 2 and 3 damping times after the NLK injection. The thin septum sheet location is indicated by the vertical black bar.

above. The beam distribution results from this tracking are shown in Fig. 11 for the first few turns and after a few damping times. In this study, 2000 particles are tracked through the accumulator ring for 30000 turns which is about 3 damping times in the horizontal direction. For the first few turns, the injected beam has a large oscillation

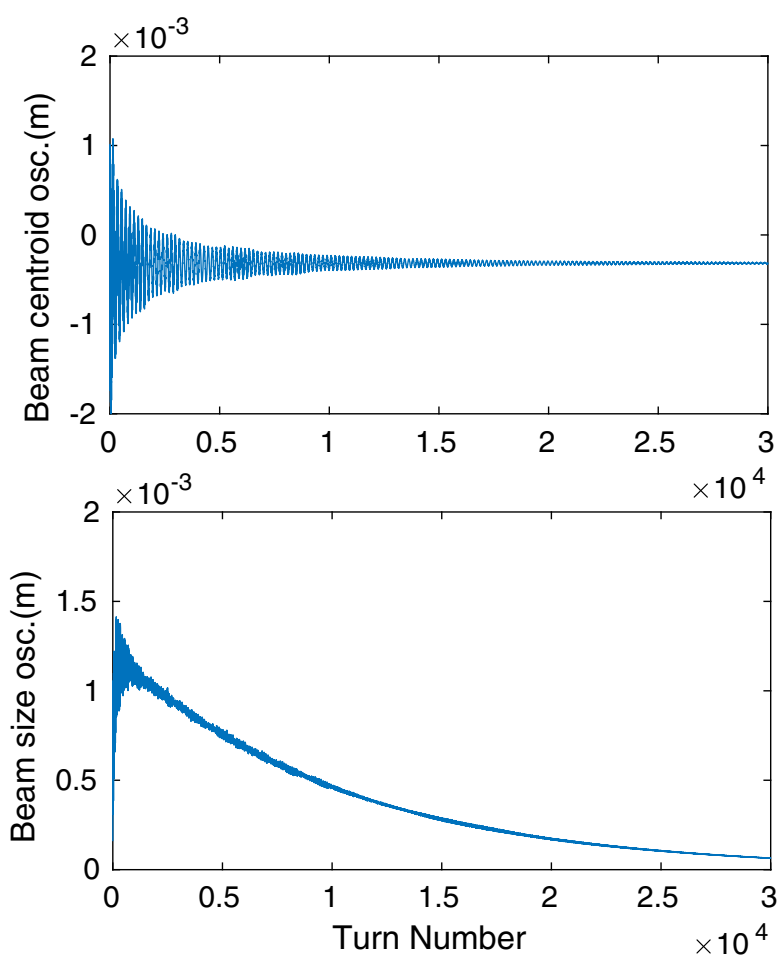

FIG. 12. Perturbations on the stored beam after the NLK injection. The upper plot is the beam centroid position and the bottom plot is the beam size oscillation. 
amplitude but is still within the dynamic acceptance of the accumulator ring. After a few damping times, the oscillation amplitude has damped down and the beam is captured by the accumulator. About 98\% injection efficiency is obtained with this tracking study, which is slightly lower than the result obtained from the optimization run because fewer particles and tracking turns are used in the MOGA run to speed up the optimization process. The injection perturbations on the stored beam are also studied by tracking stored beam (2000 particles) for several damping times. The beam centroid position and beam size are calculated at the end of each turn and results are shown in Fig. 12. As shown in the figure, the maximum oscillation amplitude of the beam centroid after the injection is about $2 \mathrm{~mm}$ which is well within the ring dynamic aperture of about $9 \mathrm{~mm}$. After 3 damping times, the oscillation has almost damped down to the closed orbit location. The beam size is increased to about $1.5 \mathrm{~mm}$ after the injection which is also within the tolerance requirement.

\section{CONCLUSIONS}

Because of its transparent injection, compactness and simplicity, the nonlinear kicker has been or will be used in several synchrotron light source facilities to inject electron beams into their storage rings. In this paper, we discussed the working principal of the NLK injection, including its magnetic field distribution, impact on the stored and injected beams as well as injection optimizations for ALS and ALS-U. The injection efficiency with a NLK can be affected by the kicker designs and injection conditions. Without optimizations, the injection efficiency could be poor, especially for a large injected beam. Using both ALS and ALS-U as examples, we have carried out comprehensive NLK injection optimizations. Although both the ALS storage ring and ALS-U accumulator ring receive the same injected beam from the ALS booster and have a similar dynamic aperture, they have different tolerance requirements of magnetic fields in the center field-free region. This consequently affects the NLK design for both ALS and ALS-U. At ALS, to reduce perturbations on the stored beam, near-zero magnetic fields at the center fieldfree region are required. In addition, to improve the injection efficiency, a narrow vertical chamber size is needed in order to have the peak field at the arriving position of the inject beam. However, at ALS-U, the perturbation on the stored beam in the accumulator is not a concern. This relaxes the tolerance requirements of magnetic fields at the center fieldfree region. With these relaxed requirements, we can move the peak field closer to the stored beam center to further improve the injection efficiency even with a relatively large vertical chamber. In either case, the maximum injection efficiency has been achieved by optimizing the NLK design and injection conditions.

\section{ACKNOWLEDGMENTS}

Authors would like to thank Peter Kuske for the discussions of the NLK tolerance requirements. This work was supported by the Office of Science, U.S. Department of Energy under DOE Contract No. DE-AC02-05CH11231.

[1] H. Ohkuma, in Proceedings of the 11th European Particle Accelerator Conference (EPAC08) (JACoW, Genoa, Italy, 2008), pp. 36-40.

[2] K. Harada, Y. Kobayashi, T. Miyajima, and S. Nagahashi, Phys. Rev. Accel. Beams 10, 123501 (2007).

[3] H. Takaki, N. Nakamura, Y. Kobayashi, K. Harada, T. Miyajima, A. Ueda, S. Nagahashi, M. Shimada, T. Obina, and T. Honda, Phys. Rev. Accel. Beams 13, 020705 (2010).

[4] T. Atkinson, M. Dirsat, O. Dressler, and P. Kuske, in Proceedings of the 2nd International Particle Accelerator Conference (IPAC2011) (JACoW, San Sebastián, Spain, 2011), pp. 3394-3396.

[5] S. C. Leemann, Pulsed multipole injection for the MAX-IV storage rings, in 5th Low Emittance Rings Workshop (ESRF, Grenoble, France, 2015).

[6] L. Liu, X. R. Resende, A. R. D. Rodrigues, and F. H. de S, in Proceedings of the 7th International Particle Accelerator Conference (IPAC2016) (JACoW, Busan, Korea, 2016), pp. 3406-3408.

[7] T. Pulampong and R. Bartolini, in Proceedings of the 4th International Particle Accelerator Conference (IPAC2013) (JACoW, Shanghai, China, 2013), pp. 2268-2270.

[8] B. MacDonald-de Neeve, M. Paraliev, and A. S. Hernandez, in Proceedings of the 8th International Particle Accelerator Conference (IPAC2017) (JACoW, Copenhagen, Denmark, 2017), pp. 3170-3173.

[9] A. White, ALS News, Vol. 394, May 29, 2018, https://als .lbl.gov/meet-leda-mother-of-light/(2018).

[10] C. Steier et al., in Proceedings of the 9th International Particle Accelerator Conference (IPAC2018) (JACoW, Vancouver, BC, Canada, 2018), pp. 4134-4137.

[11] S. C. Leemann, Phys. Rev. Accel. Beams 15, 050705 (2012).

[12] P. Kuske, Gradient specification for nonlinear injection kicker (private communication).

[13] W. H. Press, S. A. Teukolsky, W. Vetterling, and B. P. Flannery, Numerical Recipes in $C$ (Cambridge University Press, Cambridge, England, 1992).

[14] K. Deb, A. Pratap, S. Agarwal, and T. Meyarivan, IEEE Trans. Evol. Comput. 6, 182 (2002).

[15] C. Sun, D. S. Robin, H. Nishimura, C. Steier, and W. Wan, Phys. Rev. Accel. Beams 15, 054001 (2012).

[16] C. Pappas, D. Robin, C. Steier, C. Sun, and C. Swenson, in Proceedings of the 6th International Particle Accelerator Conference (IPAC2015) (JACoW, Richmond, VA, USA, 2015), pp. 1837-1839.

[17] P. Amstutz, M. P. Ehrlichman, T. Hellert, S. C. Leemann, C. Sun, C. Steier, and M. Venturini, Accumulator ring injection options and beam physics studies, Technical Note ALSU-AP-TN-2019-03. 